\title{
PREDAÇÃO E REMOÇÃO DE SEMENTES DE CINCO ESPÉCIES DE PALMEIRAS POR Guerlinguetus ingrami (Thomas, 1901) EM UM FRAGMENTO URBANO DE FLORESTA ATLÂNTICA MONTANA ${ }^{1}$
}

\author{
Luci Ferreira Ribeiro², Luciana Onécia Machado Conde ${ }^{3}$ Marcelo Tabarelli ${ }^{4}$
}

\begin{abstract}
RESUMO - Em um fragmento urbano de Floresta Ombrófila Densa Montana no Estado do Espírito Santo (Sudeste do Brasil), foram avaliados o padrão de frugivoria e as proporções de remoção, predação e armazenamento de frutos por Guerlinguetus ingrami, em relação a cinco espécies de palmeira (Syagrus pseudococos, S. ruschiana, Bactris setosa, Polyandrococos caudescens e Euterpe edulis). As espécies de Arecaceae enquadram-se na síndrome associada à dispersão por G. ingrami, na qual as espécies de plantas apresentam alta produção, grandes frutos com poucas sementes envolvidas por endocarpos resistentes e que não são usadas por outros predadores de sementes arborícolas. Os resultados apontaram que existem diferenças no padrão de frugivoria da espécie G. ingrami quando comparadas com as de espécies com a mesma síndrome; aquelas que possuem frutos maiores apresentaram maior taxa de remoção e de armazenamento de seus diásporos. E, devido à especificidade exibida por G. ingrami na atividade de dispersão de sementes, este roedor deverá atuar apenas em trocas compensatórias específicas em pequenos fragmentos defaunados. Portanto, a seletividade de G. ingrami poderia indicar que o seu papel como dispersor em pequenos fragmentos estaria restrito em função da maior probabilidade de mortalidade associada às plantas preferencialmente dispersas por essa espécie, e sua atuação como predador de sementes deve ser quantificada para que os seus efeitos, em pequenos fragmentos, sejam mais bem compreendidos.
\end{abstract}

Palavras-chave: Guerlinguetus ingrami, Frugivoria e Floresta Atlântica.

\section{PREDATION AND REMOVAL OF SEEDS FROM FIVE SPECIES OF PALMS BY Guerlinguetus ingrami (Thomas, 1901) IN AN URBAN FRAGMENT OF THE MONTANE ATLANTIC FOREST}

\begin{abstract}
This study investigated the fruvigory pattern of Guerlinguetus ingrami, considering the removal, predation and caching of the fruits of palm species (Syagrus pseudococos, S. ruschiana, Bactris setosa, Polyandrococos caudescens and Euterpe edulis) in an urban forest fragment of the montane Atlantic forest in the state of Espírito Santo, Southeastern Brazil. These palm species show characteristics theoretically expected to be seen in plants dispersed by squirrels (trees with a high productivity and whose fruits are large, with few seeds covered by rigid endocarps and that are not utilized by other arborean seed predators). Here we describe differences in the frugivory pattern exhibited by G. ingrami towards plants with the same dispersal syndrome. The diaspores of species with larger fruits were removed and cached by the squirrel at rates higher than the palms with small fruits. As a result of the high specificity showed by the squirrel's activities related to seed dispersal, this rodent should be important in compensatory shifts only in small, defaunated forest fragments. Therefore, the selectivity of G. ingrami could indicate that its role as a seed dispersor into small forest fragments would be restricted, due to the grater likelihood of mortality associated to the plants scattered by this species. The role of G. ingrami as a seed predator must be quantified for the effects of its behavioral activities in small fragments to be understood.
\end{abstract}

Keywords: Guerlinguetus ingrami, Frugivory e Atlantic forest.

\footnotetext{
${ }^{1}$ Recebido em 11.01.2008 e aceito para publicação em 02.03.2010.

2 Universidade Federal da Bahia, UFBA, Brasil. E-mail: <ribeiro.luz3@gmail.com>.

${ }^{3}$ Graduação pela Escola de Ensino Superior São Francisco de Assis, Brasil.

${ }^{4}$ Universidade Federal de Pernambuco, UFPE, Brasil.E-mail: <mtrelli@ufpe.br>.
} 


\section{INTRODUÇÃO}

A grande maioria das palmeiras possui dispersão de sementes mediada por animais, sendo estes mamíferos e, na maioria das vezes, aves (ZONA e HENDERSON, 1989). Suas sementes e frutos são um importante recurso alimentar para muitos invertebrados e vertebrados, e algumas espécies são consideradas como recursoschave para frugívoros (PERES, 1994; TERBORGH, 1986).

Os mamíferos associados à dispersão de espécies de palmeiras se enquadram em duas categorias, (1) os de maior biomassa, em que estão incluídos os grandes roedores (Agouti, Dasyprocta e Myoprocta), primatas (Cebus e Ateles) e ungulados (Tayassu e Tapirus); e (2) os pequenos redores: Scatterhoarders (Proechimys e Sciurus) (PASCHOAL e GALETTI, 1995; BORDIGNON e MONTEIRO-FILHO, 2000; JANSEN e FORGET, 2001; SILVAeTABARELLI, 2001; SVENNING 2001; WRIGHT e DUBER, 2001; ALVARENGA, 2003; FRAGOSO et al., 2003; SILVIUS e FRAGOSO, 2003; PIMENTEL e TABARELLI, 2004).

Guerlinguetus ingrami é um roedor do tipo Scatterhoarder, que armazena seus suprimentos em depósitos espalhados dentro da sua área de vida e possui, portanto, comportamento essencial para a dispersão e recrutamento de espécies vegetais (HENRY, 1999). Porém, diferentemente de outros roedores Scatterhoarders, os esquilos realizam dispersão primária (retirada dos frutos na copa da árvore) e são mais flexíveis em relação à sua dieta (GAUTIER-HION et al., 1985). De acordo com Paschoal e Galetti (1995), aparentemente os sciurídeos neotropicais são especializados em poucas espécies de plantas com grande safra anual e com sementes duras que não são usadas por outros predadores de sementes arborícolas. Todavia, poucos são os trabalhos relacionados à importância do esquilo como dispersor de sementes em florestas neotropicais. Alguns autores citaram esses roedores apenas como predadores de sementes, sem nenhuma relação com o deslocamento efetivo de diásporos (GALETTI et al., 1999; JANSEN e FORGET, 2001; SILVIUS e FRAGOSO, 2002). Porém, trabalhos em ambientes defaunados na floresta Atlântica sugerem que o esquilo atua de forma decisiva no recrutamento de algumas espécies arbóreas (BORDIGNON e MONTEIRO-FILHO, 2000; SILVA e TABARELLI, 2001; PIMENTEL e TABARELLI, 2004).

Este artigo descreve a preferência de manipulação e de enterramento, efetuados por Guerlinguetus ingrami L., dos diásporos de uma comunidade de palmeiras encontrada em fragmento urbano de 7 ha de Floresta Ombrófila Densa Montana. Analisou-se a possibilidade de que os frutos e sementes de uma comunidade de palmeiras (com diversificação, entre espécies, no tamanho da semente, na época de frutificação e na estratificação) sejam diferencialmente manipulados e predados por G. ingrami. Se tal hipótese for confirmada, G. ingrami se caracterizaria como sujeito modelador da composição e da diversidade em pequenos fragmentos defaunados.

\section{MATERIAL E MÉTODOS}

\section{1. Área de estudo}

Este trabalho foi realizado em um fragmento urbano de Floresta Atlântica Montana na região Centro-Serrana do Estado do Espírito Santo, pertencente à área do Museu de Biologia Prof. Mello Leitão (MBML). O fragmento está localizado na sede do Município de Santa Teresa (1956’12”' S, 40³5’28’’W), o qual está inserido no domínio geomorfológico do Escudo Cristalino Brasileiro e faz parte do complexo da serra da Mantiqueira (XAVIER-Da-SILVA e CARVALHO-FILHO, 1992). O município apresenta relevo montanhoso e fortemente ondulado. Os solos predominantes são classificados como Latossolo Vermelho-Amarelo distrófico (TABACOW, 1992). A floresta pode ser classificada como Floresta Ombrófila Densa Montana, uma das formações que compõem a Floresta Atlântica Brasileira (VELOSO et al., 1991). O clima do município é, segundo Köpen, do tipo subtropical úmido, sem estiagem (Cfa), com temperatura do mês mais quente superior a $22^{\circ} \mathrm{C}$ e do mês mais frio entre $3^{\circ}$ e $18^{\circ} \mathrm{C}$. A precipitação média anual está em torno de $1.868 \mathrm{~mm}$, sendo os períodos entre novembro e janeiro e entre maio e agosto relacionados às estações mais e menos chuvosas, respectivamente (MENDES e PADOVAN, 2000).

O MBML possui aproximadamente 7,7 ha. Nessa área podem ser delineados, grosso modo, três setores de acordo com a topografia da área: o vale (Setor I) e a encosta, na qual podem ser distinguidas na vertente norte (Setor II) e na vertente sul (Setor III) (CHAMAS e FERNANDES, 1995). Esses setores apresentam gradientes de altitude que variam de 670 a $750 \mathrm{~m}$, e o total de área florestada nessas vertentes é de 4,3 ha. Nesse fragmento, G. ingrami é o único roedor Scatterhoarder encontrado (RIBEIRO - Observação pessoal). Este estudo foi realizado na área de vale, Seto I, do Museu de Biologia Mello Leitão. 


\subsection{Estrutura e organização da comunidade arbórea}

As áreas vegetadas eram circundadas por residências e plantações de café e eucalipto, não possuindo conexões com outros remanescentes de mata encontrados na sede do município. As espécies arbóreas com grandes sementes se restringiam a apenas sete espécies de palmeiras (Syagrus pseudococos (Raddi) Glassman, S. ruschiana (Raddi) Glassman, S. romanzoffiana (Chamisso) Glassman, Bactris setosa Mart., Attalea oleifera Barb. Rodr., Euterpe edulis Mart. e Polyandrococos caudescens (Mart.) Barb. Rodr., estando seis dessas espécies, apesar de serem nativas (da floresta Atlântica do Estado do Espírito Santo) presentes apenas por terem sido cultivadas na área de visitação (Setor I), e a sétima espécie foi encontrada apenas no Setor III e possuía ocorrência espontânea (A. oleifera). As seis primeiras (ocorrentes no Setor I) foram aqui enfocadas.

\subsection{Espécies de palmeiras}

Henderson (2002) distinguiu dois grupos distintos de palmeiras neotropicais, no que se refere a padrões de predação e dispersão. O primeiro seria representado pelos gêneros pertencentes à tribo Cocoeae (Syagrus, Polyandrococos, Bactris), cujas espécies apresentam grandes frutos castanhos e, ou, opacos, emitem cheiros quando maduros, com mesocarpo e endosperma com maior concentração de gorduras e baixa porcentagem de carboidratos e endocarpo espesso. O outro grupo seria representado pelas espécies do gênero Euterpe (Tribo Areceae), possuindo frutos pequenos, vermelhos ou purpúreo-escuro, não apresentando cheiro na maturação, mesocarpo e endosperma com baixa proporção de gorduras e alta proporção de carboidratos, com endocarpo delgado e fibroso. Segundo Henderson, o primeiro grupo atrairia a dispersão de mamíferos e o segundo, a dispersão por aves. As espécies amostradas no pátio do MBML compreenderam esses dois grupos.

\subsection{Cocoeae}

Syagrus pseudococos (Raddi) Glassman - palmeira

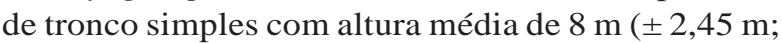
$\mathrm{N}=10)$ e perímetro de $46,7 \mathrm{~cm}( \pm 11,68 \mathrm{~cm} ; \mathrm{N}=10)$. Apresenta estípulas axilares marrom-tomentosas e infrutescência glabra. Frutos com $6,11 \mathrm{~cm}( \pm 0,11 \mathrm{~cm}$; $\mathrm{N}=100) \times 0,25 \mathrm{~cm}( \pm 0,01 ; \mathrm{N}=100)$, possuindo alargamento em direção ao ápice, que é lobado. Mesocarpo seco e endocarpo de $0,8 \mathrm{~cm}( \pm 0,08 \mathrm{~cm} ; \mathrm{N}=100)$ e lenhoso (RIBEIRO - Observação pessoal).
Syagrus ruschiana (Raddi) Glassman - Palmeira com caules múltiplos, de 2 a $8 \mathrm{~m}$ de altura e de 5 a $15 \mathrm{~cm}$ de diâmetro. Inflorescências formadas por 40 a 70 ramos floríferos finos e longos, protegidas por espata estreita e alongada. Frutos subglobosos, com cerca de 2,5 cm de comprimento, com mesocarpo fibroso e suculento (LORENZI et al., 1996).

Polyandrococos caudescens (Mart.) Barb. Rodr.Palmeira de tronco único, de 4 a $8 \mathrm{~m}$ de altura e 12 a $18 \mathrm{~cm}$ de diâmetro. Frutos globosos compressos, com cerca de 4,5 cm de comprimento, de polpa suculenta e fibrosa (LORENZI et al., 1996).

Syagrus romanzoffiana (Chamisso) Glassman Embora esteja presente na área de amostragem, esta espécie não frutificou durante o período de coleta de dados (março/2001-maio/2002).

Bactris setosa Mart. - Palmeira de caules agrupados, com 2 a 6 m de altura e 3 a 4 cm de diâmetro, com presença de espinhos. Frutos com 1 a 1,5 cm de comprimento, com epicarpo negro vinhático (LORENZI et al., 1996).

\subsection{Areceae}

Euterpe edulis Mart. - Palmeira de tronco simples, de até $20 \mathrm{~m}$ de altura. Frutos com aproximadamente $1 \mathrm{~cm}$ de comprimento, negros ou violáceos durante a maturação (LORENZI et al., 1996).

Essas espécies formam um grupo ecológico, no que se refere à oferta de frutos para a comunidade de mamíferos frugívoros, e possuem oferta diferenciada em relação ao tamanho desses frutos. Esta investigação foi baseada no gradiente de tamanho de frutos oferecidos por essas espécies, do maior (disponibilizado por $S$. pseudococos) para o menor (disponibilizado por E. edulis).

\subsection{Guerlinguetus ingrami}

Guerlinguetus ingrami L. (Rodentia, Sciuridae) é um mamífero diurno escansorial. São animais primariamente frugívoros/granívoros, mas que utilizam também na sua dieta fungos, além de outras partes vegetais, como casca de árvores e flores (BORDIGNON e MONTEIRO-FILHO, 1999).

\subsection{Fenologia das palmeiras}

Durante um período de dois anos (março/2001 a fevereiro/2003), foram realizados censos quinzenais para avaliar as épocas de frutificação das espécies estudadas, as quais se encontravam distribuídas ao longo dos 3 ha da área de vale (Setor I).

Revista Árvore, Viçosa-MG, v.34, n.4, p.637-649, 2010 


\subsection{Caracterização da população de Guerlinguetus ingrami na área de estudo}

Com o objetivo de reconhecer o tamanho populacional, proporção sexual e diferenças morfométricas e utilização de recursos relacionados ao sexo, foi caracterizada a população residente de $G$. ingrami no MBML. Os dados referentes a essa população foram obtidos entre os meses de março e outubro/2001. Durante 10 dias consecutivos por mês, foi realizada a atividade de captura e marcação dos indivíduos, sendo utilizadas 27 armadilhas do tipo Tomahawk, dispostas no solo e a $2 \mathrm{~m}$ de altura do solo, espalhadas de forma a abranger toda a área de vale. As armadilhas continham iscas com coco (Cocos nucifera, Arecaceae), abacate (Persea americana, Lauraceae) e banana (complexo relacionado à Musa paradisiaca, Musaceae). A atividade tinha início ao amanhecer (entre 04h30 e 05 h), e, ao final da tarde (entre $16 \mathrm{~h} 30$ e $17 \mathrm{~h}$ ), as armadilhas eram fechadas.

Os indivíduos capturados foram sexados, pesados e medidos. Além disso, foi realizada a observação sobre o estágio reprodutivo das fêmeas, através da dilatação abdominal e lactação, a partir do que foi inferido o período reprodutivo da espécie. Após a descrição dos dados biométricos e reprodutivos, os indivíduos foram marcados através de uma coleira flexível formada por bolinhas de metal, na qual eram inseridas duas marcas coloridas (pequenas bolas de plástico ou acrílico), sendo uma cor indicativa do sexo e a outra usada para individualização dos espécimes. Ao longo de oito meses, o esforço de captura totalizou 3.240 horas por mês.

\subsection{Padrão de frugivoria de G. ingrami}

Os dados sobre o padrão de frugivoria de G. ingrami foram obtidos a partir do método ad libitum (ALTMANN, 1974). As observações foram feitas do amanhecer (05h20) ao entardecer (18 h), obedecendo ao hábito diurno da espécie e totalizando 15 amostragens de $10 \mathrm{~h}$ contínuas cada uma, em um período de sete meses (novembro/2001 a maio/2002). O acompanhamento das atividades do esquilo foram realizadas com o auxílio de binóculo (Tasco Compact Zoom - 17 m x 25 mm) e um gravador portátil. A partir dessa metodologia, foi possível obter resultados sobre o padrão de manipulação dos frutos das espécies de palmeiras e estimar as respectivas taxas de predação e armazenamento dos seus diásporos efetuadas por G. ingrami.
A área na qual os experimentos foram realizados se restringiu a 3 dos 7 ha, estando localizada na parte administrativa e de visitação (área de vale, Setor I), a qual possuía área verde com espécies nativas e introduzidas (árvores frutíferas, palmeiras e plantas ornamentais). A partir da metodologia aplicada, os dados foram analisados enfocando o padrão de manipulação de G. ingrami para as espécies de Arecaceae, em frutificação, encontradas no local (S. pseudococos, S. ruschiana, P. caudescens, B. setosa e E. edule).

\subsection{Análise estatística}

Para determinar a independência $\left(\mathrm{H}_{0}\right)$ ou associação entre a frequência de visitação e o número de frutos armazenados, por G. ingrami, das diferentes espécies arbóreas (com tamanhos de frutos diferentes), foi utilizado o teste G (SOKAL e ROHLF, 1995; ZAR, 1996). O mesmo tipo de análise foi realizada para o número de frutos predados. Os dados biométricos (tamanho do corpo, cabeça e cauda) de machos e fêmeas de G. ingrami foram comparados entre si através de One-Way Anova, seguida de teste de Fisher. Os testes citados foram executados pelos programas Statistica 5.0 (STATSOFT, 1995) e Systat 5.0.

\section{RESULTADOS}

\subsection{Fenologia}

Durante os meses de novembro/2001 a maio/2002, foi acompanhado o período de frutificação das seis espécies de palmeiras nativas. Duas das seis espécies (S. pseudococos e P. caudescens) apresentaram frutificação no período de maior precipitação (novembrojaneiro); três delas (S. ruschiana, B. setosa e E. edule) frutificaram em um período intermediário entre o período de maior precipitação e o mais seco na região de estudo (fevereiro-abril); e uma única espécie (S. romanzoffiana) frutificou no período mais seco do ano (maio-agosto). $S$. romanzoffiana frutificou apenas durante os meses de junho-agosto/2002 (Figura 1).

\subsection{Caracterização da população de Guerlinguetus ingrami na área de estudo}

Com esforço de captura de 3.240 h por mês ao longo de oito meses foram capturados 14 indivíduos de G. ingrami no pátio do MBML, compreendendo sete fêmeas adultas, cinco machos adultos e dois machos juvenis. O comprimento médio do corpo, nas fêmeas 


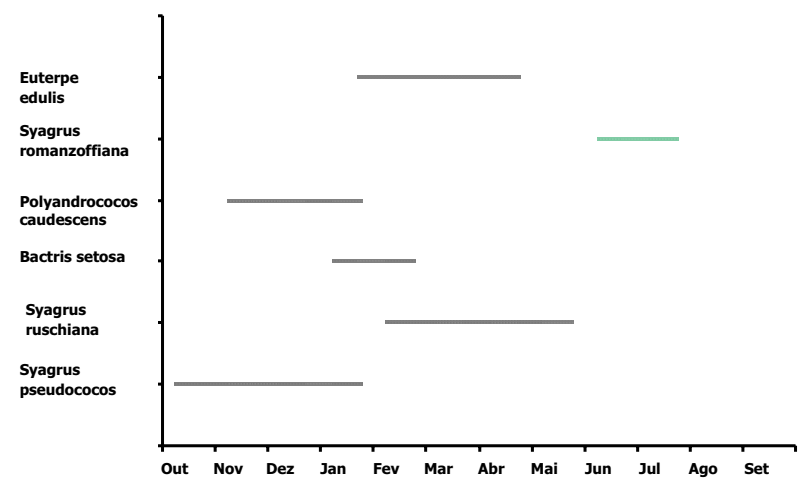

Figura 1 - Período de frutificação das espécies de palmeiras no Setor I do Museu de Biologia Prof. Mello Leitão (fragmento urbano em Santa Teresa, ES), durante o período de março de 2001 a fevereiro de 2003. Syagrus romanzoffiana frutificou apenas no ano de 2002, e as demais apresentaram frutificação nos dois anos e durante os mesmos períodos.

Figure 1 - Fruiting period of the palm species at Sector I (MBML, urban forest fragment at Santa Teresa county, Espírito Santo state, Southeastern Brazil), during March 2001-February 2003. All species fructified in both years and over the same periods, but Syagrus romanzoffiana (only at 2002).

adultas, foi de 18,33 cm ( $\pm 1,9 \mathrm{~cm} \mathrm{DP} ; \mathrm{N}=7)$; o da cauda foi de $18,9 \mathrm{~cm}( \pm 0,37 \mathrm{~cm} ; \mathrm{N}=7)$; e o peso médio foi de 242,6 g ( $\pm 2,5 \mathrm{~g} ; \mathrm{N}=5)$. As fêmeas em gestação $(\mathrm{N}=2)$ pesaram, em média, $23,25 \%$ a mais do que a média referente ao período não reprodutivo. Os machos não apresentaram diferença significativa no que se refere ao tamanho do corpo e à cauda, em relação às mesmas medidas realizadas nas fêmeas; porém, estas apresentaram peso significativamente maior que o dos machos $(\mathrm{F}=191,26$; g.l. $=1,2 ; p<0,001)$, o qual foi de 207,5 g ( \pm 5 g; $N=5)$.

Na primeira quinzena de setembro, foi presenciada a atividade de perseguição de acasalamento (cf. THOMPSON, 1977; BORDIGNON EMONTEIRO-FILHO, 1997) e, no mês de outubro, capturadas duas fêmeas gestantes.

\subsection{Padrão de frugivoria}

Durante as atividades de captura e marcação, foi realizado um levantamento preliminar da atividade comportamental de G. ingrami, enfocando seu padrão de frugivoria $(\mathrm{N}=600 \mathrm{~h}$ ). A partir desse acompanhamento preliminar, foi possível estabelecer diferença clara entre os padrões de visitação aos recursos de ambos os sexos. Os machos apresentaram baixa frequência de visitação, sendo visualizados com pouca regularidade no ambiente (nunca o mesmo macho foi visualizado em dias consecutivos na mesma fonte de recurso). Além disso, suas atividades não foram tão ricas e padronizadas, em relação à frugivoria, quando comparadas com as das fêmeas, apresentando rotas de deslocamento variáveis, visitas aos recursos pouco previsíveis e predominância da atividade de alimentação em vez da de enterramento (a qual foi rara). Em contrapartida, as fêmeas visitaram assiduamente as árvores com frutos, gastando parte das suas atividades diárias no armazenamento destes. Além disso, o comportamento observado em fêmeas levou a caracterizar a alta fidelidade no uso de rotas específicas ao longo da área amostrada, incluindo a utilização dos mesmos pontos de entrada e saída, em relação aos locais com árvores em frutificação. O comportamento altamente padronizado relacionado à atividade diária das fêmeas facilitou o acompanhamento e detalhamento das suas atividades, tornando previsível, inclusive, seus horários de alimentação.

O acompanhamento tinha início às 05h30, com a procura de indivíduos da população de G. ingrami; o primeiro indivíduo visualizado era acompanhado durante todo o restante do dia. No total, foram acompanhados três indivíduos da população, sendo todos do sexo feminino (seleção baseada nas informações citadas anteriormente).

Durante os sete meses de acompanhamento foram registradas 11 espécies na dieta de G. ingrami: $S$. pseudococos, S. ruschiana, P. caudescens, B. setosa, E. edule (Arecaceae); Inga edulis (Leguminosae); Eugenia cauliflora e Psidium sp. (Myrtaceae); Ficus citrifolia (Moraceae); Livistona chinensis (Arecaceae); e Persea americana (Lauraceae). As duas últimas são espécies exóticas, e as restantes têm registro na Mata Atlântica; porém, todas foram plantadas no local de estudo. Das 11 espécies, quatro tiveram suas sementes predadas e seus diásporos armazenados: $S$. pseudococos, $S$. ruschiana, $P$. caudescens e B. setosa. O recurso alimentar utilizado por $G$. ingrami, nas outras espécies, foi somente a polpa dos frutos (Tabela 1).

Nas observações efetuadas durante o acompanhamento das espécies de Arecaceae ( $S$. pseudococos, S. ruschiana, P. caudescens, B. setosa e E. edulis), foi observado comportamento territorialista, por parte dos indivíduos focais, resultando em atos

Revista Árvore, Viçosa-MG, v.34, n.4, p.637-649, 2010 
Tabela 1 - Descrição do padrão de frugivoria exibido por G. ingrami em relação aos diásporos consumidos no Museu de Biologia Prof. Mello Leitão (Santa Teresa, ES).

Table 1 - Frugivory pattern showed by G. ingrami in relation to the consumed diaspores at MBML (Santa Teresa county, Espírito Santo state, Southeastern Brazil).

\begin{tabular}{|c|c|c|c|c|c|c|c|}
\hline Espécies & $\begin{array}{l}\text { Poleiro de } \\
\text { despolpa }\end{array}$ & $\begin{array}{l}\text { Poleiro de } \\
\text { alimentação }\end{array}$ & $\begin{array}{l}\text { Poleiro } \\
\text { ausente }\end{array}$ & $\begin{array}{c}\text { Armazenamento } \\
\text { acima do solo }\end{array}$ & $\begin{array}{c}\text { Armazenamento } \\
\text { no solo }\end{array}$ & Polpa & Semente \\
\hline \multicolumn{8}{|l|}{ Arecaceae } \\
\hline Syagrus pseudococos & $\mathrm{X}$ & $\mathrm{X}$ & & $\mathrm{X}$ & $\mathrm{X}$ & & $\mathrm{X}$ \\
\hline Syagrus ruschiana & & $\mathrm{X}$ & & $\mathrm{X}$ & $\mathrm{X}$ & & $\mathrm{X}$ \\
\hline Poliandrococos caudescens & $\mathrm{X}$ & $\mathrm{X}$ & & $\mathrm{X}$ & $\mathrm{X}$ & & $\mathrm{X}$ \\
\hline Bactris setosa & & & $\mathrm{X}$ & $\mathrm{X}$ & $\mathrm{X}$ & & $\mathrm{X}$ \\
\hline Euterpe edulis & & & $\mathrm{X}$ & & & $X$ & \\
\hline $\begin{array}{l}\text { Livistona chinensis* } \\
\text { Mimosaceae }\end{array}$ & & & $\mathrm{X}$ & & & $\mathrm{X}$ & \\
\hline $\begin{array}{l}\text { Inga edulis } \\
\text { Myrtaceae }\end{array}$ & & & $\mathrm{X}$ & & & $\mathrm{X}$ & \\
\hline Eugenia cauliflora & & & $\mathrm{X}$ & & & $\mathrm{X}$ & \\
\hline $\begin{array}{l}\text { Psidium } s p . \\
\text { Moraceae }\end{array}$ & & & $\mathrm{X}$ & & & $\mathrm{X}$ & \\
\hline $\begin{array}{l}\text { Ficus citrifolia } \\
\text { Lauraceae }\end{array}$ & & & $\mathrm{X}$ & & & $\mathrm{X}$ & \\
\hline Persea americana & & & $\mathrm{X}$ & & & $\mathrm{X}$ & \\
\hline
\end{tabular}

*Esta espécie ocorre na área de estudo e, apesar de serem mostrados dados referentes a ela, ela não foi incluída em nenhuma das análises por se constituir numa espécie exótica.

* Although this species occurs at sudied area and its data have been collected, they were not included in analysis, since it is an exotic species.

de perseguição e vocalização, diante da presença de outras fêmeas e machos. Em raras ocasiões, pôde ser observada a presença de machos entre as fêmeas, próximos à copa de árvores em frutificação, embora a presença de filhotes de ambos os sexos fossem frequentemente tolerados pelas fêmeas (mesmo que sem relação parental direta). O comportamento agonístico tornava-se menos evidente quando da utilização das espécies arbóreas cuja fonte de recursos era relacionada à polpa do fruto e não à semente. Em árvores com grande copa, como Inga edulis e Persea americana, foram observadas mais de uma fêmea, ou fêmeas e machos, forrageando juntos.

\subsection{Padrão de manipulação dos frutos relacionada ao consumo de polpa}

Nas espécies em que somente a polpa foi consumida por G. ingrami, os frutos foram manipulados e descartados na própria planta, proporcionando efeito semelhante à sombra de sementes (proveniente da queda natural dos frutos). Apenas em F. citrifolia o fruto foi totalmente consumido. Em Psidium sp. (araçaúna), houve predação de frutos verdes e, em uma ocasião, foram registrados sete indivíduos se alimentando de frutos verdes, ao mesmo tempo e em um mesmo local.

Revista Árvore, Viçosa-MG, v.34, n.4, p.637-649, 2010

\subsection{Padrão de manipulação dos frutos com predação de sementes e armazenamento}

Syagrus pseudococos - Baseado nos dados de observação, a manipulação dos frutos seguiu o seguinte padrão: (1) inspeção dos frutos na copa das árvores e no solo, sendo a inspeção inicial a mais detalhada e as inspeções seguintes, as mais objetivas; (2) despolpa - após a coleta do fruto, a despolpa ocorria em poleiros especificamente utilizados para esse fim; (3) destino dos diásporos - após a despolpa, o destino dos diásporos pôde ser dividido em três categorias: (3.1) os frutos foram removidos para poleiros de alimentação; e os frutos que não eram predados podiam ser (3.2) armazenados acima do solo, em galhos de árvores ou moitas de palmeira ou (3.3) enterrados individualmente.

Polyandrococos caudescens - O padrão de manipulação dos frutos desta espécie foi semelhante ao descrito na espécie anterior.

Syagrus ruschiana - A partir dos dados, a manipulação dos frutos seguiu o seguinte padrão: (1) inspeção dos frutos na árvore e no solo, sendo a inspeção inicial a mais detalhada e as inspeções seguintes, as mais objetivas; (2) despolpa - após a coleta do fruto, 
a despolpa era feita na própria árvore; (3) destino dos diásporos - após a despolpa, o destino dos diásporos pôde ser dividido em três categorias: (3.1) os frutos foram removidos para poleiros de alimentação (foram identificados três poleiros), os quais foram usados por cinco indivíduos diferentes; e os frutos que não eram predados podiam ser (3.2) armazenados acima do solo, em galhos de árvores ou moitas de palmeira ou (3.3) enterrados individualmente.

Bactris setosa - Nesta espécie, observaram-se o mesmo padrão de inspeção e a ausência de poleiros de despolpa, como em S. ruschiana; e, diferentemente das demais, os frutos, após a despolpa, eram predados no mesmo local ou armazenados próximos ao recurso.

Em relação às taxas de permanência de $G$. ingrami nas espécies de Arecaceae, bem como suas taxas de enterramento, pôde-se observar que o padrão de manipulação dos diásporos de S. pseudococos (maior tamanho de fruto) foi marcadamente diferente em relação ao das demais espécies, apresentando tempo gasto na atividade de predação, de enterramento e de permanência muito mais elevado (Figura 2). Apesar de condizente com a hipótese de que tempo maior é gasto na espécie com a maior oferta de recurso, é importante destacar que esses dados podem estar sendo influenciados pelas diferenças na abundância das espécies estudadas.

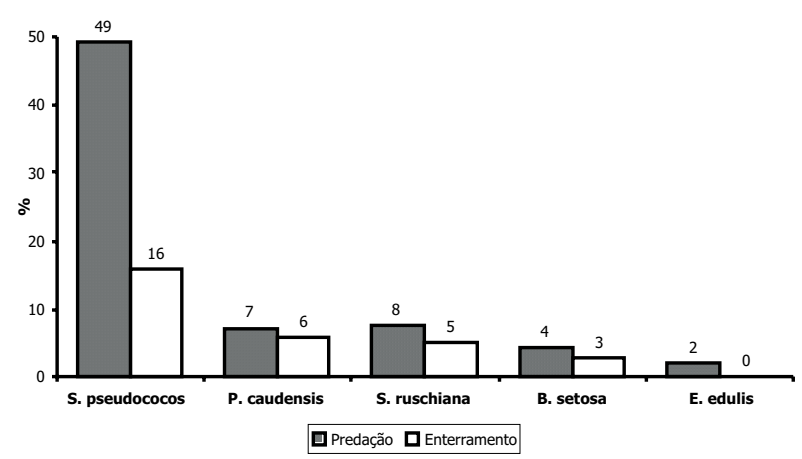

Figura 2 - Tempo de permanência e percentagem de tempo gasta em predação e armazenamento, por G ingrami, dos diásporos das espécies de Arecaceae do Museu de Biologia Prof. Mello Leitão (Santa Teresa, ES). Da esquerda para a direita, as espécies estão posicionadas de acordo com gradiente decrescente no tamanho dos frutos ( $\mathrm{N}=9.000$ minutos $)$.

Figure 2 - Predation and caching of the diaspores of palm species at MBML (Santa Teresa county, Espírito Santo state, Southeastern Brazil). The comuns refer to spent time and proportion of time directed to predation, by Guerlinguetus ingrami. From left to right, the species are positioned in accordance to a decreasing gradient in fruitsize ( $N=9,000$ minutes).
Ocorrem 14 indivíduos de S. pseudococos na área de vale, enquanto $P$. caudescens e $S$. ruschiana estão representados por apenas um indivíduo, e $B$. setosa apresenta agrupamento com oito fustes. Há cerca de 70 indivíduos de E. edulis na área de vale. Mesmo assim, tal abundância não pareceu influenciar os tempos de permanência de G. ingrami, já que essa espécie de palmeira recebeu a menor proporção de tempo gasto na manipulação dos frutos. Aparentemente, o grande número de indivíduos não compensou o pequeno tamanho dos frutos. Portanto, as análises relativas ao tempo de predação e de enterramento incluíram os dados referentes a $P$. caudescens, S. ruschiana, $B$. setosa e E. edulis. Já que a maior abundância de $S$. pseudococos poderia mascarar a preferência potencialmente alta de G. ingrami pelos seus grandes frutos, os dados dessa espécie não foram considerados nas análises.

Nas análises referentes às taxas de enterramento, além de S. pseudococos, os dados relativos a E. edulis não foram considerados, já que seus frutos ou sementes não foram enterrados por G. ingrami (Figura 3).

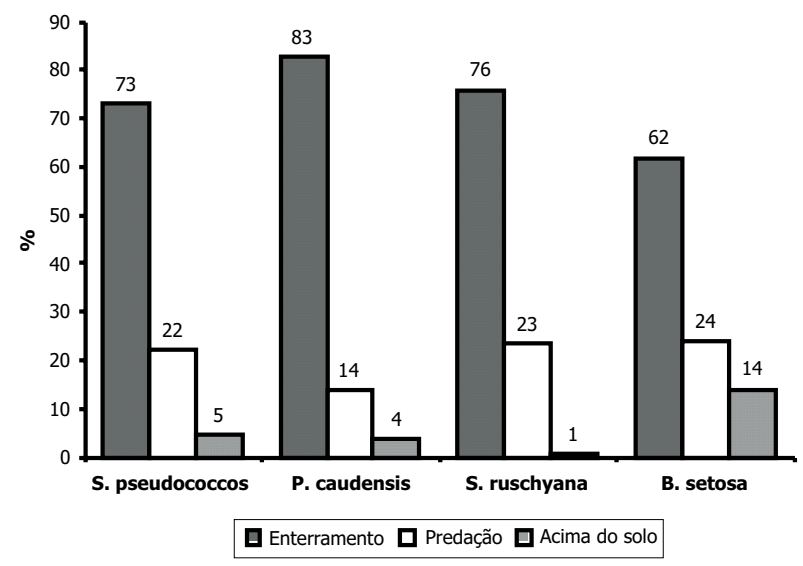

Figura 3 - Taxas de predação e armazenamento, efetuadas por G. ingrami, dos frutos das espécies de Arecaceae do Museu de Biologia Prof. Mello Leitão (Santa Teresa, ES). A sequência das espécies reflete gradiente decrescente no tamanho dos frutos ( $\mathrm{N}=961$ frutos $)$.

Figure 3 - Predation and caching rates, by Guerlinguetus ingrami, of the fruits of palm species at $M B M L$ (Santa Teresa county, Espírito Santo state, Southeastern Brazil). From left to right, the species are positioned in accordance to a decreasing gradient in fruit size ( $N=961$ fruits).

Revista Árvore, Viçosa-MG, v.34, n.4, p.637-649, 2010 
Durante as 150 h de observação, foi verificada interação de G. ingrami com as espécies de Arecaceae em 50,81\% (4.573,70 min) do tempo. Em relação ao total de horas de acompanhamento de $G$ ingrami, foi verificada diminuição significativa $(\mathrm{G}=107,07$; g.l. $=3$; $p<0,001)$ no tempo de permanência em relação a um gradiente de tamanho de frutos, do maior fruto (P. caudescens) para o menor (E. edulis) (Figura 2).

\subsection{Taxas de predação e armazenamento}

Em relação ao destino dos frutos (predação, enterramento e armazenamento acima do solo), foi encontrada diminuição significativa na taxa de enterramento ao longo do gradiente de tamanho do fruto $(G=13,05$; g.l. = 2; $p<0,001)$. Apesar de os dados de $S$. pseudococos serem mostrados, para efeitos de comparação (Figura 3), tal espécie, como discutido, não foi considerada na análise estatística. Mesmo com a inclusão dos dados referentes a essa espécie, não houve diferença significativa entre as taxas de predação das espécies de Arecaceae

$$
\text { ( } \mathrm{G}=1,06 ; \text { g.l. }=3 ; p>0,999) \text {. }
$$

\section{DISCUSSÃO}

Foi comprovado que o tempo de permanência e a proporção de enterramento de diásporos são maiores em espécies com frutos maiores, da mesma forma que a comparação entre espécies que apresentaram a síndrome de dispersão para G. ingrami (sensu RIBEIRO, 2004). Portanto, existe preferência clara, da parte de G. ingrami, por frutos maiores, a qual pode ser justificada pela qualidade do recurso. Embora a taxa de predação se tenha mostrado semelhante em todas as espécies, essa preferência por tipo de recurso traz vantagens para determinadas espécies vegetais, já que a taxa de enterramento é maior nas espécies que disponibilizam frutos maiores, o que pode se reverter em maiores taxas de germinação e recrutamento para essas espécies vegetais.

A preferência alimentar de G. ingrami está de acordo com as predições do modelo de forrageamento para roedores Scatterhoarders, em que a dispersão ótima de alimentos escondidos se encontra baseada no custo-benefício relacionado ao espaçamento entre esses esconderijos (locais de armazenamento). Nesse modelo, o benefício do armazenamento de alimentos é o produto do conteúdo energético do alimento e da probabilidade de que este sobreviva, e a probabilidade de esses locais de armazenamento permanecerem intactos aumenta com o incremento da distância entre eles, já que, dessa forma, é reduzida a oportunidade de outro animal pilhar esse recurso. Portanto, a estratégia seria direcionada a alimentos mais nutritivos e a maiores distâncias entre os locais de enterramento desse recurso (STAPANIAN e SMITH, 1978, 1984). Espécies com grandes sementes geralmente possuem alto valor nutritivo e, consequentemente, tendem a apresentar densidade menor, resultado da atividade de dispersores Scatterhoarders, em comparação com as espécies com sementes menores (STAPANIAN e SMITH, 1984; VANDER WALL, 1995). Portanto, a hipótese de que dentro de um mesmo grupo ecológico (espécies com oferta de recurso semelhante) a taxa de armazenamento de diásporos é maior em frutos com maior tamanho foi corroborada. Além disso, pôde ser observada associação entre o tempo de permanência de G. ingrami nas diferentes espécies de palmeiras e o padrão de manipulação dos diásporos, o que sugere adaptação adicional nas estratégias comportamentais de interação plantadispersor exibidas pelo esquilo.

Nas espécies com maior oferta de recurso (frutos maiores), G. ingrami passa mais tempo nas atividades de frugivoria, e o padrão comportamental se torna mais complexo. Pode-se pensar, então, que a probabilidade de ser predado seria maior justamente durante a alimentação sobre essas espécies de planta. Nesse contexto, as diferenças no padrão de manipulação dos frutos com diferentes tamanhos (e com diferenças nos tempos de permanência) podem ser interpretadas como estratégias de escape à predação. Dessa forma, não seria surpreendente que os frutos maiores, os quais demandam maior tempo de permanência e de manipulação, tenham sido despolpados em poleiros distantes da fonte do recurso, enquanto os frutos de menor tamanho tenham sido manipulados (despolpa e alimentação) na própria árvore.

Tal padrão comportamental, alternativamente, pode ser interpretado como estratégia construída como forma de não chamar a atenção de competidores em potencial para a fonte de recursos de alta recompensa. Tais hipóteses, se justificadas, não seriam mutuamente excludentes. 
Animais que exercem rotina de atividade diária podem fornecer informações de uso de recursos para outros animais (GALEF e GIRALDEAU, 2001). Smythe (1970), por exemplo, afirmou que o som emitido pela atividade de abertura dos frutos por Dasyprocta punctata (cutia) atraíram outras cutias e também predadores para esparsos locais ricos em recursos. Tais pistas e sinais, subprodutos do engajamento em atividades de sustentação à vida, são a base de quase todos os casos conhecidos de aprendizado social em vertebrados sobre quando, onde, o que e como comer (GALEF e GIRALDEAU, 2001).

Tais hipóteses reforçariam a ideia de que o padrão comportamental associado ao tamanho do recurso oferecido é determinante sobre a preferência exibida pelo agente dispersor, o que, por sua vez, atuaria diretamente sobre o sucesso reprodutivo dos indivíduos pertencentes a espécies com frutos grandes.

\subsection{Guerlinguetus ingrami como dispersor primário e despolpador de frutos}

Segundo Galetti et al. (1999), mamíferos são raramente observados consumindo frutos de E. edulis, e os referidos autores observaram que $96 \%$ das visitas aos frutos dessa espécie foram realizadas por aves (Turdinae, Rhamphastidae, Cotingidae, Cracidae e Psitacidae) e apenas 4\% por G. ingrami. Nesse caso, o esquilo atuaria como dispersor ou predador de copa (primário), assim como as espécies de aves; porém, como afirmaram os citados autores, existem indícios de que outros mamíferos (paca, cutia, cateto, veado e anta) consumam sementes de E. edulis no chão da floresta.

As observações referentes à atividade de alimentação de G. ingrami, a partir de E. edulis, se referem à predação de sementes; porém, as observações realizadas neste trabalho se restringem ao consumo de polpa. Dentro da classificação feita por Reis e Kageyama (2000), G ingrami teria comportamento-padrão de dispersor primário, semelhante aos derrubadores e despolpadores arborícolas. Nesse caso, o esquilo serviria como desperdiçador de sementes (ou como visitante oportunista), descartando-se os frutos despolpados sob a planta-mãe (MCKEY, 1975). Considerando que o número de sementes manipuladas pelos esquilos, por visita, é grande (entre 20 e 50 frutos) e existe fidelidade ao indivíduo visitado (observação pessoal para o MBML e a Estação Biológica de Santa
Lúcia, Santa Teresa, ES), a interferência no sucesso reprodutivo desses indivíduos de E. edulis, resultante da visita de esquilos, pode ser representativa.

G. ingrami apresenta o mesmo comportamento (despolpador) para outras espécies presentes no MBML, como jabuticabeira (Eugenia cauliflora, Myrtaceae), ingazeiro (Inga edulis, Mimosaceae), palmeira-de-leque chinesa (Livistona chinensis, Livistona chinensis), palmeira-bambu (Dypsis lutences, Arecaceae), clúsia (Clusia sp., Clusiaceae) e figueira (Ficus citrifolia, Moraceae), bem como na Estação Biológica de Santa Lúcia, como Eugenia sp. (Myrtaceae) e Pouteria sp. (Sapotaceae). Em todas essas espécies em que ele consome apenas polpa, não ocorreu remoção ou enterramento (CONDE e RIBEIRO, em prep.; observação pessoal).

\subsection{Guerlinguetus ingrami como dispersor primário, secundário e predador de sementes}

Nas demais espécies de palmeiras acompanhadas neste estudo, o esquilo atuou como predador de sementes. A visita de $G$. ingrami já havia sido registrada em espécies dos gêneros Syagrus e Bactris (PASCHOAL e GALETTI, 1995; SILVA e TABARELLI, 2001); apenas o gênero Polyandrococos não apresenta qualquer trabalho descritivo sobre a interação planta-dispersor.

S. romanzoffiana é citado como um dos principais itens na dieta alimentar de G. ingrami atuando como responsável pela delimitação da área de vida dessa espécie de esquilo, bem como na escolha de locais de construção de ninhos (PASCHOAL e GALETTI, 1995; ALVARENGA, 2003). Na área de estudo, as duas espécies de Syagrus, juntamente com $P$. caudescens, são os itens em que G. ingrami investe mais tempo, apresenta padrão comportamental mais complexo e sobre o qual realiza maior predação e atividade de armazenamento.

Quanto a B. setosa, é relevante ressaltar a diferença no padrão de manipulação dos frutos e a maior porcentagem de frutos armazenados acima do solo. Tal armazenamento é de curta duração (com o fruto consumido no mesmo dia ou semana) e é menos dispendioso no que se refere ao tempo gasto e à energia requerida para efetivá-lo. Esse padrão de forrageamento pode refletir o baixo investimento de G. ingrami nessa espécie de palmeira com sementes menores que as demais.

Revista Árvore, Viçosa-MG, v.34, n.4, p.637-649, 2010 
A divisão da atividade de frugivoria de G. ingrami em despolpador de frutos e predador de sementes confirma a hipótese de Henderson (2002) de que os frutos das espécies da Tribo Cocoeae apresentariam características associadas à dispersão por mamíferos, enquanto as da tribo Areceae estariam associadas a aves. Já que os frutos em que o esquilo consome apenas polpa não é realizado o deslocamento seguido de armazenamento. Essa distinção é o indicativo de que o padrão de frugivoria do esquilo beneficia apenas algumas espécies de palmeiras (grandes frutos e endosperma rico em gorduras, conf. HENDERSON, 2002), o que caracteriza essa espécie como sujeito modelador da composição e diversidade de pequenos fragmentos defaunados.

Somado aos fatores anteriormente descritos, o aumento de densidade de G. ingrami em pequenos fragmentos (Passamani com.pess.) pode interferir diretamente no sucesso reprodutivo de árvores que, embora apresentem síndrome de dispersão para esse roedor, possuam, comparativamente com as outras espécies, sementes pequenas em que o recurso seja exclusivamente constituído de polpa. Além disso, em função da baixa proporção de deslocamento e enterramento de frutos, G. ingrami pode atuar de forma pouco representativa no recrutamento de algumas espécies de palmeiras das quais são utilizadas as sementes como recurso e cujo investimento, no que se refere ao padrão comportamental de manipulação dos frutos, seja relativamente baixo.

Resultados recentes indicam que G. ingrami possui seletividade na realização da atividade de armazenamento, a qual somente é efetivada para plantas das quais são utilizadas sementes como recurso e nas quais o esquilo apresenta diferenças na atividade de manipulação, realizando maiores proporções de enterramento em espécies com frutos maiores (RIBEIRO, 2004). Outros trabalhos, ainda, apresentam essa espécie de roedor como principal dispersor em fragmentos de floresta Atlântica defaunados (BORDIGNON e MONTEIRO-FILHO, 2000; SILVA e TABARELLI, 2001; PIMENTEL e TABARELLI, 2004). As espécies de plantas estudadas nesses trabalhos apresentam síndrome de dispersão correspondente à estabelecida para G. ingrami (RIBEIRO, 2004), o que reforça, ainda mais, os resultados aqui encontrados.
Todos os dados indicam que, embora G. ingrami apresente dieta generalizada (fungos, insetos, polpa de frutos, sementes, folhas secas), essa espécie é seletiva na atividade de armazenamento e manipulação de frutos e, portanto, mesmo que ele possa visitar e consumir frutos de espécies onde os dispersores efetivos se encontram ausentes, ele não necessariamente deverá atuar como dispersor de todas essas espécies. Nesse caso, G. ingrami irá atuar em trocas compensatórias específicas, em pequenos fragmentos defaunados, e atuará, provavelmente, como predador de sementes para grande espectro de espécies, o que pode acarretar em perda de diversidade arbórea em médio ou em longo prazo.

Na literatura especializada, existem algumas predições relacionadas a espécies de plantas com grandes sementes. Sementes maiores estariam associadas à guildas de espécies arbóreas persistentes (ou tolerantes ao sombreamento em estágios sucessionais mais avançados), e suas sementes apresentariam maior longevidade, maior período de dormência e maior concentração de compostos secundários (ou precursores) do que as sementes menores, típicas de espécies pioneiras. No que se refere às suas plântulas, sementes maiores crescem em condições de grande sombreamento, acumulam proporcionalmente mais tecido de reserva e podem ser mais resistentes à herbivoria (através da translocação de compostos secundários das sementes para as plântulas) (FOSTER e JANSON, 1985; FOSTER, 1986). Espécies com sementes maiores apresentam, portanto, adaptações biológicas relacionadas à estrutura física e biológica de ambientes localizados em interior de mata madura ou com baixo nível de perturbação. Assim, poder-se-ia classificar G. ingrami como dispersor típico em florestas maduras. Porém, a sua aparente resistência à fragmentação e à caça o torna a principal espécie citada como dispersor efetivo em fragmentos defaunados (BORDIGNON e MONTEIRO-FILHO, 2000; SILVA TABARELLI, 2001; PIMENTEL e TABARELLI, 2004). Florestas tropicais fragmentadas apresentam modificações físicas e biológicas, em comparação com florestas contínuas, o que resulta em mortalidade e perda de diversidade de espécies arbóreas, principalmente no que se refere a árvores de dossel e de grandes sementes (TABARELLI et al., 2004). Portanto, a seletividade de G. ingrami poderia indicar que o seu papel como dispersor em pequenos fragmentos estaria restrito, em função da maior probabilidade de mortalidade associada às plantas 
preferencialmente dispersas por essa espécie, e sua atuação como predador de sementes deve ser quantificada para que os efeitos da sua atuação, em pequenos fragmentos, sejam mais bem compreendidos.

\section{REFERÊNCIAS}

ALTMANN, J. Observational study of behavior: sampling methods. Behaviour, v.49, p.227-267, 1974.

\section{ALVARENGA, C. A. Estudo de uma população de Sciurus ingrami Thomas, 1901 (Rodentia, Sciuridae) na Reserva Particular do Patrimônio Natural Serra do Caraça, Minas Gerais, Brasil. Dissertação de Mestrado. Curso de Pós-Graduação em Zoologia dos Vertebrados, Pontifícia Universidade Católica, Belo Horizonte, MG, 2003. \\ BORDIGNON, M.; MONTEIRO-FILHO, E. L.A. Comportamentos e atividades diárias de Sciurus ingrami (Thomas) em cativeiro (Rodentia, Sciuridade). Revista Brasileira de Zoologia, v.14, n.3, p.707-722, 1997.}

BORDIGNON, M.; MONTEIRO-FILHO, E. L.A. Seasonal food resourse of the squirrel Sciurus ingrami in a secondary Araucaria Forest in Southern Brazil. Studies on Neotropical Fauna \& Environment, v.34, n.3, p.137-140, 1999.

BORDIGNON, M.; MONTEIRO-FILHO, E. L. A. O serelepe Sciurus ingrami (Sciuridae: Rodentia) como dispersor do Pinheiro do Paraná Araucaria angustifolia (Araucariaceae: Pinophyta).

Arquivos de Ciência Veterinária, v.3, n.3, p.139-144, 2000.

CHAMAS, C. C.; FERNANDES, H. Q. B. Relatório Manejo do Parque do Museu de Biologia Professor Mello Leitão: Aspectos de Estética, Funcionalidade e Segurança. Relatório Técnico Interno MBML, 1995.

CHIARELLO, A. G. Effects of fragmentation of the Atlantic forest on mammal communities in southeastern Brazil. Biological Conservation, v.89, n.7, p.71-82, 1999.

CLARK, D. B.; CLARCK, D. A.; READ, J. M. Edaphic variation and mesoscale distribution of tree species in a neotropical rainforest. Journal of Ecology, v.86, n.1, p.101-112, 1998.
CONDIT, R. Research in large, long-term tropical forest plots. Trends in Ecology \& Evolution, v.10, n.1, p.18-22, 1995.

CONNELL, J. H. On the role of natural enemies in preventing competitive exclusion in some marine animals and in rain forest trees. In: BELER, P. J.; GRADWELL, G. R (Eds.). Dynamic

Populations. Proceedings of Advanced Study Institute on Dynamics of Numbers in Populations. Oosterbeek, 1970, Wageningen: Centre for Agricultural Publishing and Documentation, 1971. pp. $298-310$.

EISENBERG, J. F.; REDFORD, K. Mammals of the Neotropics: the Central

Neotropics. Vol. 3 Ecuador, Peru, Bolivia, Brazil. Chicago: University of Chicago Press, 1999.

EMMONS, L. H.; FEER, F. Neotropical rainforest mammals: A field guide. $2^{\mathrm{a}}$ ed. Chicago: University of Chicago Press, 1997.

FORGET, P.-M. Scatterhoarding of Astrocaryum paramaca by Proechymis in French Guiana: comparison with Myoprocta exilis. Tropical Ecology, v.94, n.7, p.255-261, 1991.

FOSTER, S. A. On the adaptive value of large seeds for tropical moist forest trees: a review and a synthesis. The Botanical Review, v.52, n.3, p.260-299, 1986.

FOSTER, S. A.; JANSON, C. H. The relationship between seed size and stablishment conditons in tropical wood plants. Ecology, v.66, n.6, p.773-780, 1985.

FRAGOSO, J. M. V.; SILVIUS, K. M.; CORREA, J. A. Long-distance seed dospersal by tapirs increases seed survival and aggregates tropical trees. Ecology, v.84, n.8, p.1998-2006, 2003.

FUNDAÇÃO SOS MATA ATLÂNTICA, INPE \& ISA. Atlas da evolução dos remanescentes florestais e ecossistemas associados no domínio da Mata Atlântica no período 1990-1995. São Paulo: Fundação SOS Mata Atlântica, Instituto Nacional de Pesquisas Espaciais e Instituto Socioambiental, 1998.

Revista Árvore, Viçosa-MG, v.34, n.4, p.637-649, 2010 
GALEF, B. G. JR.; GIRALDEAU, L.-A. Social influences on foraging in vertebrates: causal mechanisms and adaptive functions. Animal Behaviour, v.61, n.1, p.3-15, 2001.

GALETTI, M.; ZIPPARRO, V. B.; MORELLATO, P. C. Fruiting phenology and fruvigory on the palm Euterpe edulis in a lowland Atlantic forest of Brazil. Ecotropica, v.5, p.115-122, 1999.

GAUTIER-HION, A.; DUPLANTIER J. M.; QURIS, R.; FEER, F.; SOURD, C.; DECOUX, J.-P.; DUBOST, G.; EMMONS, L.; ERARD, C.; HECKETSWEILER, P.; GENTRY, A. H. 1985. Dispersal ecology and diversity in neotropical forest communities. Naturwiss v.7, p.303-314, 1985.

GRUBB, P. J. The maintenance of speciesrichness in a plant communities: the importance of the regeneration niche. Biology Review, v.52, n.9, p.107-145, 1977.

HARMS, K. E.; POWERS, J. S.; MONTGOMERY, R. A. Variation in small sapling density, understory cover, and resource availability in four neotropical forests. Biotropica, v.36, n.3, p.40-51, 2004.

HENDERSON, A. Evolution and ecology of palms. New York: The New York Botanical Garden Press, 2002. 259p.

HENRY, O. Frugivory and the importance of seeds in the diet of the orange-rumped agouti (Dasyprocta leporina) in French Guiana. Journal of Tropical Ecology v.15, p.291-300, 1999.

JANSEN, P. A.; FORGET, P.-M. Scatterhoarding rodents and tree regeneration. In: BONGERS, E. (Ed.). Dynamics and plant-animal interactions in a neotropical rainforest. Amsterdam: Kluwer Academic Publishers, 2001. p.275-288.

LORENZI, H.; SOUZA, H. M.; MEDEIROSCOSTA, J.; CERQUEIRA, L. S. C.; VON BEHR, N. Palmeiras do Brasil: Nativas e Exóticas. São Paulo: Editora Plantarum, 1996.

MCKEY, D. Seed dispersal. In: GILBERT, L. E.; RAVEN, P. H. (Eds.). Coevolution of Animal and Plants, 1975.
MENDES, S. L.; PADOVAN, M. P. A Estação Biológica de Santa Lúcia, Santa Teresa, Espírito Santo. Boletim do Museu de Biologia Mello Leitão, v.7, n.11/12, p.24-29, 2000.

PASCHOAL, M.; GALETTI, M. Seasonal food use by the Neotropical squirrel Sciurus ingrami in Southeasthern Brazil. Biotropica, v.27, n.1, p.268-273, 1995.

PERES, C. A. Composition, density, and fruiting phenology of arborescent palms in an Amazonian terra firme forest. Biotropica, v.26, n.3, p.285-294, 1994.

PIMENTEL, D. S.; TABARELLI, M. Seed dispersal of the palm Attalea oleifera in a remnant of the Brazilian Atlantic Forest. Biotropica, v.36, n.1, p.74-84, 2004.

REIS, A.; KAGEYAMA, P. Y. Dispersão de sementes do palmiteiro (Euterpe edulis Martius Palmae). In: Reis, M. S.; Rris, A. (Eds.). Euterpe edulis Martius (palmiteiro): biologia, conservação e manejo. Itajaí: Herbário Barbosa Rodrigues, 2000. p.60-92.

RIBEIRO, L. F. Dispersão e predação de grandes sementes por Guerlinguetus ingrami L. em fragmentos de floresta Atlântica montana no estado do Espírito Santo, Brasil. Tese de Doutorado. Programa de PósGraduação em Biologia Vegetal, Universidade Federal de Pernambuco (UFPE), Recife, PE, 2004.

SILVA, J. M. C.; TABARELLI, M. Tree species impoverishment and the future flora of the Atlantic forest of northeast Brazil. Nature, v.404, n.3, p.72-73, 2000.

SILVA, M.G.; TABARELLI, M. Seed dispersal, plant recruitment and spatial distribution of Bactris acanthocarpa Martius (Arecaceae) in a remnant of Atlantic forest in northeast Brazil. Acta Oecologia, v.22, n.5-6, p.259-268, 2001.

SILVIUS, K. M.; FRAGOSO, J. M. V. Pulp handling by vertebrate seed dispersers increases palm seed predaton by bruchid beetles in the Northern Amazon. Journal of Ecology v.90, n.6, p.1024-1032, 2002.

SILVIUS, K. M.; FRAGOSO, J. M. Red-rumped Agouti (Dasyprocta leporina) home range use in an Amazonian forest: implications for the aggregated distribution of forest trees. Biotropica, v.35, n.1, p.74-83, 2003. 
SMYTHE, N. Relationships between fruiting seasons and seed dispersal methods in a neotropical forest. American Naturalist, v.104, n.935, p.25-35, 1970.

SOKAL, R. R.; ROHLF, F. G. Biometry. New York: Freeman, 1995.

STAPANIAN, M. A.; SMITH, C. C. A model for scatterhoarding: coevolution of fox squirrels and black walnuts. Ecology, v.59, n.10, p.884-896, 1978.

STAPANIAN, M. A.; SMITH, C. C. Densitydependent survival of scatterhoarded nutz: an experimental approach. Ecology, v.65, n.8, p.1387-1396, 1984.

STATSOFT. STATISTICA for Windows [Computer program manual]. StatSoft, Tulsa, OK, 1995.

SVENNING, J.-C. On the role of microenvironmental heterogeneity in the ecology and diversification of neotropical rain-forest palms (Arecaceae). The Botanical Review, v.67, n.1, p.1-53, 2001a.

SVENNING, J.-C. Environmental heterogeneity, recruitment limitation and the mesoscale distribution of plams in a tropical montane rain forest (Maquipucuna, Ecuador). Journal of Tropical Ecology, v.17, p.97-113, 2001b.

TABACOW, J. Proposta de zoneamento ambiental para o município de Santa Teresa. Monografia de Especialização, Universidade Federal do Espírito Santo (UFES), Vitória, ES, 1992.

TABARELLI, M.; SILVA, J. M. C.; GASCON, C. Forest fragmentation, synergisms and the impoverishment of neotropical forests. Biodiversity and Conservation, v.13, n.7, p.1419-1425, 2004.

TERBORGH, J. Keystone plant resource in tropical forest. In: SOULÉ, M. E. (Ed.).

Conservation biology: The science of scarcity and diversity. Sunderland: Sinauer, 1986.

THOMPSON, D. C. Reproductive behaviour of grey squirrel. Canadian Journal of Zoology, v.55, p.1176-1184, 1977.

VANDER WALL, S. B. The effects of seed value on the caching behavior of yellow pine chipmunks. Oikos, v.74, p.533-537, 1995.
VELOSO, H. P.; RANGEL-FILHO, A. L. R.; LIMA, J. C. A. Classificação da vegetação brasileira adaptada a um sistema universal. Rio de Janeiro: IBGE, 1991.

VIEIRA, L. A. Caracterização da avifauna do parque do Museu de Biologia Professor Mello Leitão, Santa Teresa, Espírito Santo. Monografia de Bacharelado. Curso de Ciências Biológicas, Escola Superior São Francisco de Assis (ESFA), Santa Teresa, ES, 2002.

WELDEN, C. W. S.; HEWETT, S. W.; HUBBELL, S. P.; FOSTER, R. B. Sapling survival, growth, and recruitment: relationship to canopy height in a Neotropical forest. Ecology, v.72, n.2, p.35-50, 1991.

WRIGHT, S. J. The dispersion of eggs by a bruchid beetle among Scheelea palm seeds and the effect of distance to the parent palm. Ecology, v.64, n.10, p.1016-1021, 1983.

WRIGHT, S. J. The myriad consequences of hunting for vertebrates and plants in tropical forests. Perspectives in Plant Ecology, Evolution and Systematics, v.6, n.1-2, p.73-86, 2003.

WRIGHT, S. J.; DUBER, H. C. Poachers and forest fragmentation alter seed dispersal, seed survival, and seedling recruitment in the palm Attalea butyraceae, with implications for tropical tree diversity. Biotropica, v.33, n.12, p.583-595, 2001.

WRIGHT, S. J.; ZEBALLOS, H.; DOMÍNGUEZ, I.; GALLARDO, M. M.; MORENO, M. C. IBÁÑEZ, R. Poachers alter mammal abundance, seed dispersal, and seed predation in a neotropical forest. Conservation Biology, v.14, n.2, p.227-239, 2000.

XAVIER-DA-SILVA, J.; CARVALHO-FILHO, L. $M$. Esboço da sinopse da evolução geológico-geomorfológica do município de Santa Teresa-ES. Relatório Técnico. Rio de Janeiro: Universidade Federal do Rio de Janeiro, 1992.

ZAR, J. H. Biostatistical analysis. New Jersey: Prentice Hall, 1996.

ZONA, S.; HENDERSON,A.A Areview of animal-mediated seed dispersal of palms. Selbyana, v.11, p.6-21, 1989.

Revista Árvore, Viçosa-MG, v.34, n.4, p.637-649, 2010 